\title{
The COVID-19 Pandemic in Senegal: Experience of an Internal Medicine Department as an Epidemic Treatment Center
}

\author{
Atoumane Faye*, Awa Cheikh Ndao, Nafissatou Diagne, Mouhamed Dieng, Maimouna Sow, \\ Baidy Sy Kane, Boundia Djiba, Abdoulaye Pouye \\ Internal Medicine Department, University Hospital Center Le Dantec, Dakar, Senegal \\ Email: ^atoufayemi@yahoo.fr, ndaoawacheikh@gmail.com, nafissatoud@hotmail.com, docdieng@gmail.com, \\ mouna89sow@hotmail.com, baidykane@gmail.com,boundiadjiba@yahoo.fr,docpouye@yahoo.fr
}

How to cite this paper: Faye, A., Ndao, A.C., Diagne, N., Dieng, M., Sow, M., Kane, B.S., Djiba, B. and Pouye, A. (2022) The COVID-19 Pandemic in Senegal: Experience of an Internal Medicine Department as an Epidemic Treatment Center. Open Journal of Internal Medicine, 12, 13-21. https://doi.org/10.4236/ojim.2022.121002

Received: November 30, 2021

Accepted: January 23, 2022

Published: January 26, 2022

Copyright () 2022 by author(s) and Scientific Research Publishing Inc. This work is licensed under the Creative Commons Attribution-NonCommercial International License (CC BY-NC 4.0). http://creativecommons.org/licenses/by-nc/4.0/ (c) (i) (8) Open Access

\begin{abstract}
Introduction: The COVID-19 pandemic has turned the global health system upside down. Senegal recorded its first case on 2 March 2020. As part of its control strategy, the internal medicine department was set up as an epidemic treatment center (ETC). We report on our experience in the management of patients hospitalized in the said center. Material and Methods: Retrospective and descriptive study with analytical aim carried out at the ETC of the CHU Le Dantec of Dakar during the period from 29 April to 30 October 2020 (1st wave) then from 30 December 2020 to 30 April 2021 (2nd wave). All hospitalized patients who tested positive for RT-PCR were included. Epidemiological, clinical, paraclinical and evolutionary data were collected from a pre-established survey form and analysed using the software. Results: Five hundred and seventy files were collected: 379 patients (66\%) in the 1st wave and 191 patients (34\%) in the 2 nd wave. There were 312 men and 258 women, i.e. a sex ratio of 1.22. The median age was 56 years [ $1.5-100$ years]. Two thirds of the patients (66\%) were over 50 years of age and 223 patients (39.1\%) were over 65 years of age. Community transmission was reported in $72 \%$ of cases. The average length of hospital stay was 10 days [1 - 32]. Clinically, the mild form predominated (50.9\%); the severe and critical forms were $32.6 \%$. Comorbidity was noted in $68 \%$ of patients. The different comorbidities were: hypertension (36.1\%), diabetes (28.2\%), advanced CKD (9.8\%). The case fatality rate was $17.2 \%$ with a mean age of 70 years. Conclusion: COVID-19 is responsible for respiratory but also systemic manifestations. This 3rd pandemic particularly affects vulnerable people with a significant morbidity and mortality, thus requiring the implementation of standardized CTE with multidisciplinary teams in the control strategies.
\end{abstract}




\section{Keywords}

Pandemic, COVID-19, Comorbidities, Lethality

\section{Introduction}

After the pandemics due to SARS-CoV-1 in 2002 in China and MERS-CoV in 2012 in the Arabian Peninsula, a 3rd pandemic due to SARS-CoV-2 has emerged in 2019 [1]. The cases broke out in December 2019 in the city of Wuhan, Hubei province, China in patients with unexplained severe pneumonia [2]. On 30 January 2020, WHO declared this viral infection a "global health emergency" and in February declared it COVID-19 (coronavirus disease 2019) [3]. The people most affected by COVID-19 were the elderly and those with comorbidities including hypertension, diabetes, cancer, HIV infection and other immunosuppressants. The African continent has not been spared by this pandemic. Indeed, Senegal recorded its first case on 2 March 2020 and since then the number of cases has not stopped increasing. As part of its strategy to combat this third pandemic, the Senegalese health authorities had set up the internal medicine department of the CHU Le Dantec as an epidemic treatment center for the care of the elderly, patients with comorbidities and pregnant women. The objective of our study was to determine the epidemiological, clinical and evolutionary aspects of patients admitted to the ETC for COVID-19.

\section{Materials and Methods}

This was a retrospective and descriptive study in the Epidemic Treatment Center (ETC) of the University Hospital Le Dantec, Dakar during the period from April 29 to October 30, 2020 (1st wave) and from December 30, 2020 to April 30, 2021 (2nd wave). All hospitalized patients for a confirmed COVID-19 RT-PCR infection on naso-pharyngeal swab or rapid test, if the patient is symptomatic with typical or comorbid scannographic involvement, were included. The data were collected on individual collection sheets and the data was entered using the SPHINX 2000 software. Data analysis and processing were performed on the EXCEL 2007 software. We studied epidemiological, clinical and evolutionary parameters. The clinical forms were divided into benign, moderate, severe and critical forms.

\section{Results}

During the study period, we collected 570 files composed of 312 men (55\%) and 258 women (45\%), i.e. a sex ratio of 1.20 . The median age of our patients was 59 years with extremes of 1.5 and 100 years. Two thirds of the patients (66\%) were over 50 years old. People over 65 years of age represented $39.1 \%$ of our study population. The majority of patients (90\%) came from the Dakar region. Seventy-two percent of patients were from community transmission. The monthly 
distribution of admissions is shown in Figure 1.

Clinically, half of the patients (50.9\%) were mild on admission. Table 1 shows the distribution of the different clinical forms.

The presence of comorbidities was noted in 390 patients (68\%). The main comorbidities were hypertension (36.1\%), diabetes (28.2\%), Chronic Kidney Disease stage $4 / 5$ (9.8\%) and heart disease (5.2\%). The average length of stay was 10 days \pm 5 days. The length of hospital stay did not exceed 14 days in $77.4 \%$ of cases. Ninety-eight patients $(17.2 \%)$ died. The average age of the deceased patients was 70 years. About 3/4 (71.4\%) of patients died were older than 65 years. Of the deceased patients, $71 \%(\mathrm{~N}=70)$ had a critical form and $26 \%(\mathrm{~N}=25)$ a severe form. There were no deaths in the mild forms.

Comparing the data from the 2 waves, 379 patients (66\%) were admitted in the first wave and 191 (34\%) in the second. The average age was 51 years with a slight female predominance (51\%) in the first wave and 64 years with $66 \%$ men in the second wave. Comorbidities were present in $68 \%$ of patients in the first wave and $71 \%$ in the second wave. $84 \%$ of patients in the first wave had a mild or moderate form. Severe and critical forms in the second wave were $74 \%$ and $78 \%$ respectively with a statistically significant variation $(\mathrm{p}=0.0001)$ and a high case fatality of $35.6 \%$ versus $7.9 \%$ in the first wave.

\section{Discussion}

In our study, the median age of our patients (59 years) was slightly higher than that of Taieb [4] in Senegal, which was 57 years, and the Chinese cohort, where the median age was 56 years [5]. Our data are close to those found in Guinea Conakry by Adamou [6] and in Algeria by Ketfi [7] who found a mean age of 59

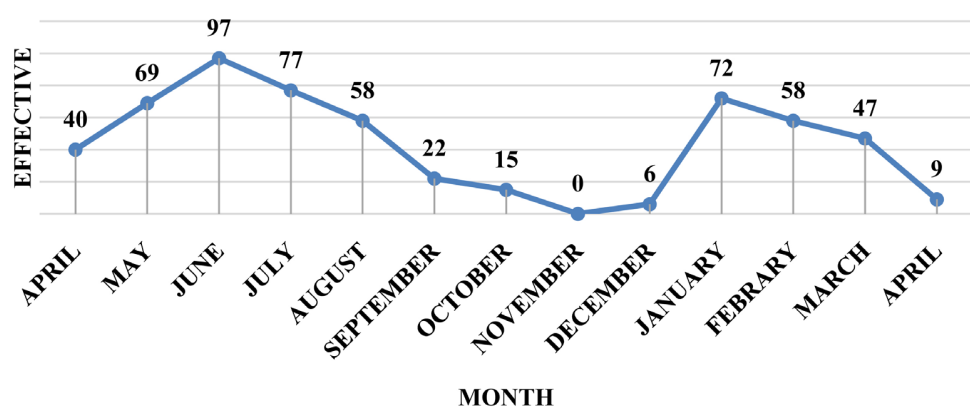

Figure 1. Monthly admission (April 2020-April 2021) of COVID-19 patients.

Table 1. Distribution of patients by clinical forms.

\begin{tabular}{ccc}
\hline Clinical forms & Effective $(\mathrm{n}=570)$ & Frequency (\%) \\
\hline Benign & 290 & 50.9 \\
Moderate & 94 & 16.5 \\
Severe & 96 & 16.8 \\
Critical & 90 & 15.8 \\
\hline
\end{tabular}


and 53 years respectively. COVID-19 infection does not spare any age, as shown by our data but also those reported in the literature. In our series, $66 \%$ of the patients were over 50 years of age and those over 65 years of age represented 39\% of our total study population. There is heterogeneity in the demographics of the reported patient populations. In the study by Ketfi [7], more than half (54.6\%) of the patients were over 50 years old. Taieb [4], in his series noted $28.6 \%$ of patients over 60 years of age, close to the series of Nachega [8] in the Democratic Republic of Congo which noted a proportion of $23.3 \%$ of subjects over 60 years of age. However, our study population was younger than that observed in Europe and the United States [9]. Moreover, the median age observed during the second wave was higher (65 years) than during the first wave because our center mainly welcomed elderly people and patients with comorbidities such as hypertension, diabetes, chronic kidney disease and pregnant women. This selection was due to the multidisciplinary nature of the center, with a haemodialysis unit, an operating theatre and a delivery room for COVID patients. During the first wave, all the people who tested positive, whether symptomatic or not, were hospitalized to avoid the spread of the virus, which explains the clear predominance of mild and moderate forms. During the second wave, hospitalization was selective.

The male predominance of SARS-COV-2 infection found in our study (55\%) was similar to the data reported in the literature, as shown by the study population of Taieb [4], which consisted of 53.6\% men, and those of Wang [5] and Sixt [10], which were $54.3 \%$ and $55.4 \%$ respectively. Our data are lower than those found by Kefti [7], which were $60.5 \%$ male, and Nachega [8], which were $65.6 \%$. In the literature, intermediate results have been reported in the work of Guan [11] and $\mathrm{Wu}$ [12] who found a sex ratio of 1.4 and 1.8 respectively. This male predominance could be related to the higher frequency of exposure and risk factors for severity in men. Table 2 summarizes the socio-demographic characteristics of our patients.

Clinically, the spectrum of COVID-19 infection ranges from asymptomatic or pauci-symptomatic forms to severe forms, characterised by acute respiratory failure or even respiratory distress requiring mechanical ventilation. After an incubation period of about five days, $70 \%$ of infected patients develop cough, fever or dyspnea [11]. This phase of viral invasion is followed, in some patients, by an inappropriate immune response marked by worsening respiratory symptoms and an inflammatory syndrome, usually eight to ten days after the first symptoms [13]. This dysimmune phase, sometimes referred to as a cytokine storm, may be associated with coagulopathy, which some authors have described as viral sepsis [14]. COVID-19 is a multifaceted disease due to its complexity. It is essentially manifested by a respiratory attack, but a richer symptomatology has been reported [15], as shown by the different manifestations observed in our series (Table 1), dominated by asthenia (74.9\%), myalgia (43\%), fever $(40.8 \%)$ and dyspnea (37.5\%). The main clinical signs are summarized in Table 3. 
Table 2. Socio-demographic characteristics of patients.

\begin{tabular}{|c|c|c|}
\hline Characteristics & Effective $(\mathrm{n}=570)$ & Frequency (\%) \\
\hline \multicolumn{3}{|l|}{ Gender } \\
\hline Female & 258 & 45 \\
\hline Male & 312 & 55 \\
\hline \multicolumn{3}{|l|}{ Age (years) } \\
\hline$<16$ & 14 & 2.4 \\
\hline$[16-35]$ & 92 & 16.1 \\
\hline$[35-50]$ & 88 & 15.4 \\
\hline$[50-65]$ & 153 & 26.8 \\
\hline$\geq 65$ & 223 & 39.1 \\
\hline \multicolumn{3}{|l|}{ Origin } \\
\hline Dakar & 513 & 90 \\
\hline Other regions & 54 & 9.5 \\
\hline Foreign Country & 3 & 0.5 \\
\hline \multicolumn{3}{|l|}{ Length of stay (days) } \\
\hline$<7$ & 150 & 26.3 \\
\hline$[7-14]$ & 291 & 51.1 \\
\hline$[14-21]$ & 112 & 19.6 \\
\hline$[21-28]$ & 15 & 2.6 \\
\hline$\geq 28$ & 2 & 0.4 \\
\hline
\end{tabular}

Table 3. Distribution of main clinical signs observed in our series.

\begin{tabular}{cccc}
\hline Clinical signs & $\mathbf{n}=\mathbf{5 7 0}$ & $\mathbf{1}^{\text {st }}$ wave $(\mathrm{n}=\mathbf{3 7 9})$ & $\mathbf{2}^{\text {nd }}$ wave $(\mathrm{n}=191)$ \\
\hline Anosmia & $90(16.8 \%)$ & $65(17.1 \%)$ & $31(16.2 \%)$ \\
Ageusia & $116(20.3 \%)$ & $81(21.4 \%)$ & $35(18.3 \%)$ \\
Headache & $191(33.5 \%)$ & $123(32.4 \%)$ & $68(35.6 \%)$ \\
Fever & $233(40.8 \%)$ & $139(36.6 \%)$ & $94(49.2 \%)$ \\
Asthenia & $427(74.9 \%)$ & $276(72.8 \%)$ & $151(79.0 \%)$ \\
Dyspnea & $214(37.5 \%)$ & $95(25.1 \%)$ & $119(62.3 \%)$ \\
Cough & $194(34.0 \%)$ & $102(26.9 \%)$ & $92(48.1 \%)$ \\
Vigilance disorders & $98(17.2 \%)$ & $57(15.0 \%)$ & $41(21.4 \%)$ \\
Chest pain & $46(8.0 \%)$ & $25(6.5 \%)$ & $21(10.9 \%)$ \\
Hemoptysis & $17(3.0 \%)$ & $11(2.9 \%)$ & $6(3.1 \%)$ \\
Diffuse pain/Aches & $245(43.0 \%)$ & $154(40.6 \%)$ & $91(47.6 \%)$ \\
Sore throat & $54(9.4 \%)$ & $39(10.3 \%)$ & $15(7.8 \%)$ \\
Nausea/vomiting & $36(6.3 \%)$ & $26(4.7 \%)$ & $10(5.2 \%)$ \\
Abdominal pain & $34(5.9 \%)$ & $21(5.5 \%)$ & $13(6.8 \%)$ \\
Diarrhea & $23(4.0 \%)$ & $12(3.1 \%)$ & $11(5.7 \%)$ \\
\hline
\end{tabular}


The most frequently reported signs in the literature are fever, cough and myalgia. This is evidenced by the study by Taieb [4] which found this triad of fever, cough and myalgia in $55.8 \%, 47.6 \%$ and $31.2 \%$ respectively. In the series by Plaçais [15], the complaints were dominated by the triad "asthenia-fever-cough". Sixt [10], in his study of 222 patients, the clinical signs most frequently found were asthenia (80.2\%), fever (76\%), cough (66\%) and dyspnea (62\%). Digestive and neurological signs have also been reported in the literature, supporting the systemic nature of the infection. In our study, the digestive signs were nausea-vomiting (6.3\%), abdominal pain (5.9\%), and diarrhea (4\%). The frequency of diarrhea ranges from $3.8 \%$ to $15 \%$ according to the studies [15]. Digestive disorders may be the first clinical sign, particularly in elderly subjects [15]. Neurological manifestations were not uncommon in the latter, as shown by the frequency of vigilance disorders (17.2\%) noted in our series, which was close to that reported by Mao's team [16], which was $14.8 \%$. The clinical expression in the elderly can be modified with a misleading symptomatology dominated by neurological or digestive signs, making the diagnosis sometimes difficult. However, in a meta-analysis of 17 studies and 1285 elderly patients included, the symptoms of COVID-19 in this population were close to those observed in young adults [17].

Comorbidities are possible risk factors for increased severity of COVID-19. The main comorbidities reported in the literature in patients hospitalized for COVID-19 were hypertension, diabetes, cardiovascular disease and cancer. Our results are in agreement with the literature. In our series, the frequency of hypertension was $36.1 \%$, diabetes (28.2\%) and chronic kidney disease $(9.8 \%)$. The frequency of comorbidities varied between series. In Taieb's study [4], the main comorbidities were hypertension (17.1\%), diabetes (14.5\%) and chronic lung disease (8\%). The frequency of hypertension, diabetes and CKD was $43 \%$, $14 \%$ and $4 \%$ respectively in the Wang D cohort [5]. In the study by Nachega [8] in the Democratic Republic of Congo, the most frequent comorbidities were hypertension (25.4\%), diabetes (14\%) and heart disease (3.9\%). The heterogeneity of the study populations explains the variability of comorbidities. Our center, which mainly accommodated the elderly and patients suffering from chronic renal disease with a dialysis unit, would explain this high frequency of diabetes, hypertension and chronic renal disease. Indeed, more than 750 hemodialysis sessions were performed in this unit specially dedicated to COVID-19 infected patients. The main comorbidities most often reported in the literature are: hypertension $(15 \%-30 \%)$, diabetes $(7.4 \%-19 \%)$ and cardiovascular diseases $(2.5 \%$ 8\%) [2] [3] [12].

The frequency of cancerous pathologies noted in our study (1.6\%) was slightly higher than those observed in the literature, which were around 0.5 [11], 0.9 [10] and 1\% [18]. However, Ketfi [7] and Wang [5] found higher frequencies of 5\% and $10 \%$ respectively.

Pregnant women represent a population at risk of infection due to the physiological changes specific to pregnancy. Fifty-four pregnant women were hospita- 
lized in the center $(9.4 \%), 16$ of whom were in the third stage of pregnancy. Of these, 2 gave birth by vaginal delivery and 2 by cesarean section for overdue delivery. There was no maternal-fetal transmission. All nasopharyngeal swabs taken from the newborn at the 24th hour were negative. However, the possibility of maternal-fetal transmission was raised by Liu [19] as three newborns of infected mothers had positive RT-PCR SARS-CoV-2 on day 2; all of them presented with a pneumopathy picture confirmed on chest CT scan. In another retrospective study conducted by Chen $\mathrm{H}$ [20], involving 9 pregnant women in their third trimester without any comorbidity, the maternal clinical, biological and radiological data were comparable to the general population. There was no severe respiratory form [20]. However, other authors have shown an increased risk of severe disease in pregnant women during the SARS-CoV and MERS CoV pandemics [21]. In our study, only one woman in the second third of her pregnancy, who was hypertensive and well-controlled, developed a critical illness that justified her transfer to the ICU. The outcome was favourable. There were no deaths among the pregnant women. These various studies, admittedly limited in terms of numbers, seem to show that pregnant women in their third year of pregnancy have a risk of developing a severe form of the disease as observed in the general population. However, the possibility of maternal-fetal transmission is still controversial [20].

The mortality rate in our series was $17.2 \%$ (98 patients). Seventy patients (71.4\%) died aged over 65 years. This high mortality was proportional to the severity of the clinical picture, age and comorbidities. The mortality rate varied between series. The $16 \%$ mortality rate noted in the study by Sixt [10] is close to that found in our series. The team of Li et al. [22], reported a lower case fatality rate; it was $8 \%$ in the age group [ $70-79$ years] and $14.8 \%$ in subjects over 80 years. In this series, patients with an unfavorable outcome were significantly older (77.7 years vs. 67.7 years, $p<0.001$ ), with more comorbidities. In our case, mortality was related to advanced age with decompensation of defects, comorbidities, severity of the clinical form at admission.

According to Bulubas et al. [23], factors that predicted COVID-19 mortality with a high level of evidence included age, smoking, cerebrovascular disease, chronic obstructive pulmonary disease (COPD), chronic kidney disease, arterial hypertension, diabetes, cardiac arrhythmia, and cancer.

\section{Limitations of the study}

The study was monocentric and retrospective with some missing data in the files not allowing us to provide all the clinical parameters. Our ETC, which houses an intensive care and reanimation unit, largely explains the significant lethality compared to other treatment centers.

Blood gas and CT scans were not routine; they were only performed in certain patients with signs of respiratory severity.

\section{Conclusion}

COVID-19 is responsible for systemic manifestations with respiratory involve- 
ment that is often revealing. The frequency of comorbidities with a high lethality in the elderly and co-morbid patients at risk of severe forms justifies the setting up of ETC with multidisciplinary teams. Thus, it is becoming urgent to prepare for possible waves in the long term and to definitively break this "panic-forget" cycle with strategies based on standard recommendations adapted to our realities and the lessons learned during the two waves.

\section{Conflicts of Interest}

The authors declare no conflicts of interest regarding the publication of this paper.

\section{References}

[1] Wong, G., Liu, W., Liu, Y., Zhou, B., Bi, Y. and Gao, G.F. (2015) MERS, SARS and Ebola: The Role of Super-Spreaders in Infectious Disease. Cell Host \& Microbe, 18, 398-401. https://doi.org/10.1016/j.chom.2015.09.013

[2] Zhu, N., Zhang, D., Wang, W., et al. (2020) A Novel Coronavirus from Patients with Pneumonia in China, 2019. The New England Journal of Medicine, 382, 727-723. https://doi.org/10.1056/NEJMoa2001017

[3] Wu, Y., Ho, W., Huang, Y., et al. (2020) SARS-Cov-2 Is an Appropriate Name for the New Coronavirus. The Lancet, 395, 949-950. https://doi.org/10.1016/S0140-6736(20)30557-2

[4] Taieb, F., Mbaye, K.F., Tall, B., Lakhe, N.A., Talla, C., et al. (2021) Hydroxychloroquine and Azithromycin Treatment of Hospitalized Patients Infected with SARSCoV-2 in Sénégal from March to October. Journal of Clinical Medicine, 10, 2954. https://doi.org/10.3390/jcm10132954

[5] Wang, D., Hu, B., Hu, C., et al. (2020) Clinical Characteristics of 138 Hospitalized Patients with 2019 Novel Coronavirus-Infected Pneumonia in Wuhan, China. JAMA, 323, 1061-1069. https://doi.org/10.1001/jama.2020.1585

[6] Danamou, J., Bangoura, A., Camara, L.M., Camara, D., Traoré, D.A., et al. (2021) Caractéristiques épidémiologiques et cliniques des patients COVID 19 admis en réanimation à l'hôpital Donka de Conakry: Etude descriptive des 140 premiers cas hospitalisés. Anesthésie \& Réanimation, 7, 102-109.

https://doi.org/10.1016/j.anrea.2021.01.001

[7] Ketfi, A., Chabati, O., Chemali, S., et al. (2020) Profil clinique, biologique et radiologique des patients Algériens hospitalisés pour COVID-19: Données préliminaires. Pan Africa Medical Journal, 35, 77. https://doi.org/10.11604/pamj.supp.2020.35.2.23807

[8] Nachega, J.B., Ishoso, D.K., Otoye, J.O., Hermans, M.P., Machekano, R.N., et al. (2020) Clinical Characteristics and Outcomes of Patients Hospitalized for COVID-19 in Africa: Early Insights from the Democratic Republic of the Congo. American Journal of Tropical Medicine and Hygiene, 103, 2419-2428.

[9] Richardson, S., Hisrch, J.S., Narasimhan, M., Crawford, J.M. and McGinn, T. (2020) Presenting Characteristics, Comorbidities and Outcomes among 5700 Patients Hospitalized with COVID-19 in the New York City Area. JAMA, 323, 2052-2059. https://doi.org/10.1001/jama.2020.6775

[10] Sixt, T., Moretto, F., Abdallahoui, M., Chavanet, P., Devilliers, H., Catherine, F. and Piroth, L. (2020) 21èmes Journées nationales d'infectiologie. Médecine et maladies 
infectieuses, 50, S62-S63. https://doi.org/10.1016/j.medmal.2020.06.120

[11] Guan, W., Ni, Z., Hu, Y., Liang, W., Ou, C., He, J., et al. (2020) Clinical Characteristics of Coronavirus Disease 2019 in China. The New England Journal of Medicine, 382, 1708-1720. https://doi.org/10.1056/NEJMoa2002032

[12] Wu, C., Chen, X., Cai, Y., et al. (2020) Risk Factors Associated with Acute Respiratory Distress Syndrome and Death in Patients with Coronavirus Disease 2019 Pneumonia in Wuhan, China. JAMA Internal Medicine, 180, 934-943.

[13] Huang, C., Wang, Y., Li, X., Ren, L., Zhao, J., Hu, Y., et al. (2020) Clinical Features of Patients Infected with 2019 Novel Coronavirus in Wuhan, China. The Lancet, 395, 497-506.

[14] Li, H., Liu, L., Zhang, D., Xu, J., Dai, H., Tang, N., et al. (2020) SARS-CoV-2 and Viral Sepsis: Observations and Hypotheses. The Lancet, 395, 1517-1520. https://doi.org/10.1016/S0140-6736(20)30920-X

[15] Plaçais, L. and Richier, Q. (2020) COVID-19: Clinical, Biological and Radiological Characteristics in Adults, Infants and Pregnant Women. An Up-to-Date Review at the Heart of the Pandemic. La Revue de Médecine Interne, 41, 308-318. https://doi.org/10.1016/j.revmed.2020.04.004

[16] Mao, L., Jin, H., Wang, M., Hu, Y., Chen, S., He, Q., et al. (2020) Neurologic Manifestations of Hospitalized Patients with Coronavirus Disease 2019 in Wuhan, China. JAMA Neurology, 77, 683-690.

https://doi.org/10.1001/jamaneurol.2020.1127

[17] Neumann-Podczaska, A., Al-Saad, S.R., Karbowski, L.M., et al. (2020) COVID-19 Clinical Picture in the Elderly Population: A Qualitative Systematic Review. Aging and Disease, 11, 988-1008. https://doi.org/10.14336/AD.2020.0620

[18] Zhou, F., Yu, T., Du, R., Fan, G., Liu, Y., Liu, Z., et al. (2020) Clinical Course and Risk Factors for Mortality of Adult Inpatients with COVID-19 in Wuhan, China: A Retrospective Cohort Study. The Lancet, 395, 1054-1062. https://doi.org/10.1016/S0140-6736(20)30566-3

[19] Li, K., Wu, J., Wu, F., Guo, D., Chen, L., Fang, Z., et al. (2020) The Clinical and Chest CT Features Associated with Severe and Critical COVID-19 Pneumonia. Investigative Radiology, 55, 327-331. https://doi.org/10.1097/RLI.0000000000000672

[20] Chen, H., Guo, J., Wang, C., Luo, F., Yu, X., Zang, W., et al. (2020) Clinical Characteristics and Intrauterine Vertical Transmission Potential of COVID-19 Infection in Nine Pregnant Women: A Retrospective Review of Medical Records. The Lancet, 395, 809-815. https://doi.org/10.1016/S0140-6736(20)30360-3

[21] Schwatz, D.A. (2020) An Analysis of 38 Pregnant Women with COVID-19, Their Newborn Infants, and Maternal Fetal Transmission of SARS-CoV-2: Maternal Coronavirus Infections and Pregnant Outcomes. Archives of Pathology \& Laboratory Medicine, 144, 799-805. https://doi.org/10.5858/arpa.2020-0901-SA

[22] Liu, Y., Chen, H., Tang, K. and Guo, Y. (2020) Clinical Manifestations and Outcome of SARS-CoV-2 Infection during Pregnancy. Journal of Infection. https://doi.org/10.1016/j.jinf.2020.02.028

[23] Bulubas, I., Vogel, T. and Muller, M. (2021) Les facteurs pronostiques dans la Covid-19. NPG Neurologie-Psychiatrie-Geriatrie. 